\title{
Expanded spectral domain-OCT findings in the early detection of hydroxychloroquine retinopathy and changes following drug cessation
}

\author{
David R. Lally ${ }^{1,2}$, Jeffrey S. Heier ${ }^{2}$, Caroline Baumal ${ }^{1}$, Andre J. Witkin', Steven Maler ${ }^{1}$, Chirag P. Shah², \\ Elias Reichel ${ }^{1}$, Nadia K. Waheed ${ }^{1}$, Igor Bussel ${ }^{1}$, Adam Rogers ${ }^{1}$ and Jay S. Duker ${ }^{1,2^{*}}$
}

\begin{abstract}
Purpose: To report expanded SD-OCT findings of HCQ retinopathy that may assist the clinician in earlier diagnosis. To characterize structural changes of HCQ retinopathy with SD-OCT after drug cessation.

Methods: Setting: Private practice and academic institution. Patient Population: Patients at New England Eye Center and Ophthalmic Consultants of Boston in Boston, MA diagnosed with HCQ retinopathy and followed after drug cessation. Retrospective clinical data review by the Boston Image Reading Center. Main Outcome Measures: SD-OCT findings suggestive of HCQ retinopathy before parafoveal ellipsoid disruption. Change in SD-OCT morphological appearance and retinal thickness of each of the nine subfields corresponding to the Early Treatment of Diabetic Retinopathy Study areas.

Results: Thirty eyes with HCQ retinopathy were followed with SD-OCT after drug cessation. Findings before disruption of the parafoveal EZ included parafoveal outer nuclear layer (ONL) thinning, disruption of the parafoveal interdigitation zone, and reduced reflectivity of the parafoveal EZ. In early toxicity, $75 \%$ developed progression after drug cessation, including disruption of the parafoveal EZ and retinal pigment epithelium and thinning of the ONL. Eyes with obvious toxicity had greater inferior outer ring thinning 12 months after drug cessation compared to early toxicity ( $p=0.002,95 \% \mathrm{Cl}-2$ to $-8 \mu \mathrm{m}$ ). In obvious toxicity, the nasal inner subfield showed more thinning than the temporal inner subfield at 12 months after drug cessation ( $p=0.018,95 \% \mathrm{Cl}-1$ to $-8 \mu \mathrm{m}$ ).
\end{abstract}

Conclusions: Once HCQ retinopathy is diagnosed and the medication is discontinued, structural retinal changes commonly occur.

\section{Background}

Hydroxychloroquine (HCQ) has been used for therapy of rheumatologic disorders since the 1950s. Ocular toxicity associated with HCQ use was initially described in the 1960s [1, 2]. The incidence of HCQ retinopathy is estimated at $1 \%$ after consumption of HCQ for 5 years [3]. It is marked by paracentral and central scotoma and

\footnotetext{
*Correspondence: jduker@tuftsmedicalcenter.org

${ }^{1}$ Vitreoretinal Service, Departments of Ophthalmology, New England Eye Center, Tufts University School of Medicine, 260 Tremont Street, Boston, MA 02116, USA

Full list of author information is available at the end of the article
}

decreased color vision. Risk factors predisposing to the development of HCQ retinopathy include a daily dose $>6.5 \mathrm{mg} / \mathrm{kg}$, cumulative dose $>1000 \mathrm{~g}$, duration of use over 5 years, elderly age, kidney or liver dysfunction, or preexisting maculopathy [4]. HCQ retinopathy is rare considering the widespread utilization of this drug; however, the retinopathy has potential to produce irreversible visual loss [1].

There is limited data regarding structural and functional changes of HCQ retinopathy after drug cessation [5-7]. Studies from the 1960s and 1970s evaluated individuals taking the more toxic, related drug chloroquine, but were 
limited to visual acuity, visual fields, and fundus examination to assess for progression of retinopathy. In addition, these studies typically evaluated patients with advanced disease who had already developed a Bull's eye fundus appearance. In the present day, the less toxic HCQ is used instead of chloroquine. The American Academy of Ophthalmology revised the screening guidelines in 2011 to include spectral domain-optical coherence tomography (SD-OCT), fundus autofluorescence (FAF), and multifocal ERG (mfERG) when available to assess the macula in patients taking HCQ [4]. The new guidelines were designed for earlier detection of HCQ retinopathy before funduscopic changes become apparent. Studies reviewing mfERG and FAF after drug cessation have demonstrated both progression and improvement in retinal function in moderate to severe toxicity [8-11]. It is unclear whether less severe cases of retinopathy progress after HCQ cessation.

$\mathrm{SD}-\mathrm{OCT}$ is a highly sensitive and reproducible imaging modality commonly used in clinical practice. SD-OCT is capable of detecting characteristic macular changes of HCQ toxicity. These typical macular abnormalities include loss of the parafoveal ellipsoid zone (EZ), parafoveal thinning of the outer nuclear layer (ONL) and inner plexiform layer (IPL), the "flying saucer" sign, and peripapillary nerve fiber layer thinning [12-14]. Few reports have examined progression of changes in SDOCT appearance after drug cessation. Marmor et al. [11] reported that SD-OCT appearance in patients with early HCQ toxicity, defined as patchy parafoveal visual field defects, did not change after drug cessation. However, moderate cases, defined as a 50-100\% parafoveal ring of damage on visual field testing, did demonstrate progressive parafoveal thinning. Kellner et al. [15] showed long-term follow-up of eyes with moderate to severe chloroquine/HCQ toxicity resulting in cystoid macular edema and epiretinal membrane formation. However, no changes were observed in early toxicity.

This study examines longitudinal SD-OCT scans after cessation of HCQ and stratifies eyes based on severity of retinopathy to evaluate whether this plays a role in structural changes. An independent reading center was used to limit observational bias in interpreting the SDOCT scans. Furthermore, SD-OCT findings are identified before clear parafoveal ellipsoid zone (EZ) disruption becomes apparent, which may enable earlier diagnosis of HCQ retinopathy.

\section{Methods}

Data collection was obtained following approval from the Internal Review Board (IRB) of Tufts Medical Center, and was in compliance with the Health Insurance Portability and Accountability Act. Research tenets were followed in accordance with the Declaration of Helsinki.
A retrospective, consecutive case series of eyes with HCQ retinopathy that were followed after drug cessation was conducted at New England Eye Center, Boston, Massachusetts and Ophthalmic Consultants of Boston, Boston, Massachusetts. The study interval was from January 1, 2006 to July 12, 2013. Eyes were included if they were diagnosed with HCQ retinopathy and had SD-OCT imaging at the time of drug cessation and on follow-up exam. In addition, eyes were included only if the followup SD-OCTs were performed on the same machineeither Cirrus HD-OCT (Carl Zeiss Meditec, Dublin, CA) or Heidelberg Spectralis HRA + OCT (Heidelberg Engineering, Germany).

HCQ retinopathy was diagnosed by detecting abnormalities attributable to HCQ on clinical examination, Humphrey 10-2 or 24-2 visual fields, SD-OCT, and/or FAF when available. Of note, all eyes in this study had visual field defects consistent with HCQ toxicity. Multifocal ERG was performed in only 8 eyes, and in these eyes, toxicity was confirmed with visual field defects and SD-OCT findings. Therefore, mfERG was not included in the analysis. Eyes were excluded if there was no clinical examination on record coincident with the time of $\mathrm{HCQ}$ cessation, if the follow up OCT was performed on a different OCT machine than at the time of drug cessation, or if time-domain OCT was performed. Ocular symptoms, best-available visual acuity, macular appearance, and imaging modalities (OCT, FAF) available at the time of HCQ retinopathy diagnosis and after HCQ cessation were recorded. The cumulative HCQ dose was calculated.

The first primary outcome measure was the morphological change in 1-line horizontal OCT scans from the time of discontinuing HCQ to the most recent examination. Vertical OCT scans were not routinely ordered by the treating physician and therefore unable to be included in analysis. If there were SD-OCT images available that demonstrated findings that preceded disruption of the parafoveal EZ, these findings were recorded and analyzed by the reading center. The second primary outcome measure was the change in retinal thickness in each of the 9 Early Treatment of Diabetic Retinopathy Study (ETDRS) OCT sub-fields at 12 months after drug cessation.

The SD-OCT scans were evaluated by two masked, trained readers from the Boston Image Reading Center (BIRC, Boston, MA). The readers were provided raw data from the cube scans, which were qualitatively assessed for alterations in the normal morphological retinal features. Readers analyzed SD-OCT images from the time of drug cessation and most recent follow up exam, and assessed whether any interval changes had occurred. After careful deliberation, common features which were most consistently recorded were decreased reflectivity of the ellipsoid zone (EZ), disruption of the EZ, disruption 
of the interdigitation zone (IZ), disruption of the retinal pigment epithelium (RPE), disruption of the external limiting membrane (ELM), and thinning of the outer nuclear layer (ONL). These characteristics were evaluated to be present or absent in both the foveal region (central $1500 \mu \mathrm{m})$ and parafoveal region $(0-2000 \mu \mathrm{m}$ from the border of the foveal region on either side). The findings were considered true findings only if both readers agreed on the finding (i.e. hyporeflective, RPE atrophy, etc.) and the location of the finding (i.e. fovea, nasal parafovea, temporal parafovea, or both nasal and temporal parafovea). In no instances was there an opposite finding on either side of the fovea, such as hyperreflectivity on the nasal side and hyporeflectivity on the temporal side.

For data analysis, the primary cohort was divided into three groups based on BIRC's grading of the 1-line horizontal OCT scan through the fovea at the time of drug cessation (Fig. 1). The purpose of the division was to separate early, obvious, and severe cases of toxicity, as the development of new screening modalities such as SDOCT has allowed for detection of earlier cases of HCQ toxicity. The Early group consisted of eyes presenting without clear disruption of both the foveal and parafoveal ellipsoid zone (EZ) as judged by BIRC's interpretation of the SD-OCT at the time of drug cessation. Fundus examination of these eyes showed early macular findings, or none at all, at the time of diagnosis. The Obvious group consisted of eyes presenting with an intact foveal EZ but a clearly disrupted parafoveal EZ on either one or both sides of the fovea as judged by the BIRC graders. These eyes clinically manifested the classic parafoveal RPE changes. The Severe group consisted of eyes presenting with clear foveal EZ disruption with associated parafoveal EZ disruption. These eyes had poor visual acuity at presentation due to foveal and parafoveal outer retinal atrophy.
Retinal thickness measurements of each of the nine subfields corresponding to the Early Treatment of Diabetic Retinopathy Study (ETDRS) areas were examined. ETDRS SD-OCT thickness measurements consist of nine separate regions, which are defined by three concentric rings centered on the fovea with diameters of 1,3 , and $6 \mathrm{~mm}$, respectively. The two outer rings are divided into quadrants by two intersecting orthogonal lines. Only eyes that underwent SD-OCT imaging at baseline and follow-up with either Cirrus HD-OCT (Carl Zeiss Meditec, Dublin, CA) or Heidelberg Spectralis HRA + OCT (Heidelberg Engineering) were included in this analysis. Eyes that had baseline Stratus OCT (Carl Zeiss Meditec, Dublin, CA) were excluded.

Statistical analysis was performed using GraphPad (GraphPad Software, Inc., La Jolla, CA USA). We used an unpaired $t$ test to compare the changes in retinal thickness between the groups, while a paired t-test was used to compare the changes within a group. $95 \%$ confidence intervals were calculated for any statistically significant changes observed as defined as $\mathrm{p}<0.05$.

\section{Results}

Thirty-six patients were identified with HCQ retinopathy. Thirteen patients were excluded because an OCT was not performed at the time of diagnosis. Eight patients were excluded for having a time domain-OCT at the time of drug cessation. Thirty eyes of 15 female patients met inclusion criteria and were followed by repeated examinations after drug cessation. Clinical features of each patient are outlined in Table 1 and in each of the three groups in Table 2. All patients were Caucasian. Half of the eyes were emmetropic, and half were ametropic (range -6.0 to +6.0 diopters). None of the patients had kidney or liver dysfunction.

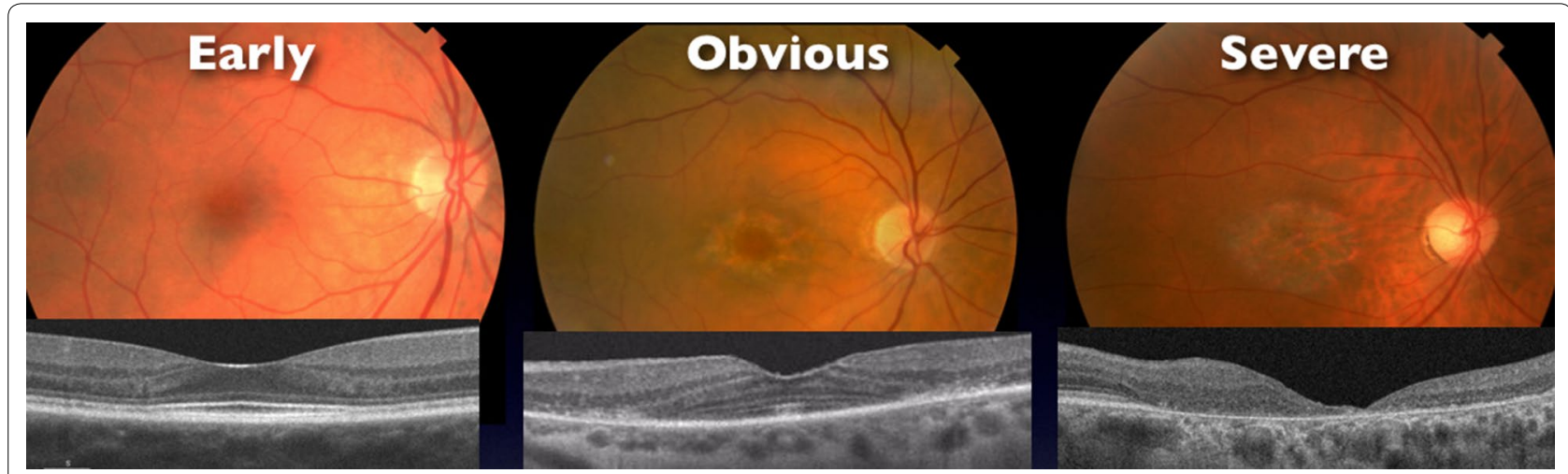

Fig. 1 Fundus photograph and SD-OCT example of each group. Cohort divided into three groups based on BIRC's grading of ellipsoid zone at the time of drug cessation. Early—no disruption of parafoveal or foveal EZ; Obvious — disruption of parafoveal EZ with intact foveal EZ; Severe—disruption of both foveal and parafoveal EZ 
Table 1 Clinical summary of patients

\begin{tabular}{|c|c|c|c|c|c|c|c|c|c|}
\hline $\begin{array}{l}\text { Patient/sex/ } \\
\text { age }\end{array}$ & $\begin{array}{l}\text { HCQ cumu- } \\
\text { lative dose } \\
\text { (g) }\end{array}$ & $\begin{array}{l}\text { Body mass } \\
\text { index }\end{array}$ & $\begin{array}{l}\text { HCQ indica- } \\
\text { tion }\end{array}$ & $\begin{array}{l}\text { Follow-up } \\
\text { after HCQ } \\
\text { cessation } \\
\text { (months) }\end{array}$ & $\begin{array}{l}\text { HCQ cessa- } \\
\text { tion VA }\end{array}$ & $\begin{array}{l}\text { Most recent } \\
\text { VA }\end{array}$ & $\begin{array}{l}\text { Symptoms } \\
\text { at HCQ ces- } \\
\text { sation }\end{array}$ & $\begin{array}{l}\text { Symptoms } \\
\text { at follow-up }\end{array}$ & $\begin{array}{l}\text { Progression } \\
\text { after HCQ } \\
\text { cessation? }\end{array}$ \\
\hline$E 1 / F / 57$ & 730 & a & $\begin{array}{l}\text { Psoriatic } \\
\text { arthritis }\end{array}$ & 13 & $\begin{array}{l}\text { OD:20/20 } \\
\text { OS:20/20 }\end{array}$ & $\begin{array}{l}\text { OD:20/20 } \\
\text { OS:20/20 }\end{array}$ & None & None & Yes (OCT, HVF) \\
\hline $\mathrm{E} 2 / \mathrm{F} / 74$ & 890 & a & SLE & 13 & $\begin{array}{l}\text { OD:20/30 } \\
\text { OS:20/40 }\end{array}$ & $\begin{array}{l}\text { OD:20/40 } \\
\text { OS:20/40 }\end{array}$ & $\begin{array}{l}\text { Decreased } \\
\text { near vision }\end{array}$ & $\begin{array}{l}\text { Decreased } \\
\text { near vision }\end{array}$ & Yes (OCT) \\
\hline $\mathrm{E} 3 / \mathrm{F} / 69$ & 584 & 51 & RA & 56 & $\begin{array}{l}\text { OD:20/25 } \\
\text { OS:20/25 }\end{array}$ & $\begin{array}{l}\text { OD:20/30 } \\
\text { OS:20/30 }\end{array}$ & None & None & $\begin{array}{l}\text { Yes (OCT, } \\
\text { HCF) }\end{array}$ \\
\hline$E 4 / F / 61$ & 438 & 26 & RA & 12 & $\begin{array}{l}\text { OD:20/25 } \\
\text { OS:20/25 }\end{array}$ & $\begin{array}{l}\text { OD:20/25 } \\
\text { OS:20/25 }\end{array}$ & Floaters & None & No \\
\hline $\mathrm{E} 5 / \mathrm{F} / 65$ & 949 & 17 & RA & 42 & $\begin{array}{l}\text { OD:20/20 } \\
\text { OS:20/20 }\end{array}$ & $\begin{array}{l}\text { OD:20/20 } \\
\text { OS:20/20 }\end{array}$ & Floaters & None & Yes (OCT, HVF) \\
\hline O1/F/48 & 2210 & a & SLE & 18 & $\begin{array}{l}\text { OD:20/30 } \\
\text { OS:20/40 }\end{array}$ & $\begin{array}{l}\text { OD:20/30 } \\
\text { OS:20/30 }\end{array}$ & None & None & Yes (OCT) \\
\hline $\mathrm{O} 2 / \mathrm{F} / 65$ & 4380 & 24 & RA & 36 & $\begin{array}{l}\text { OD:20/20 } \\
\text { OS:20/20 }\end{array}$ & $\begin{array}{l}\text { OD:20/20 } \\
\text { OS:20/20 }\end{array}$ & Floaters & Floaters & Yes (OCT) \\
\hline O3/F/62 & 1022 & 24 & RA & 42 & $\begin{array}{l}\text { OD:20/50 } \\
\text { OS:20/60 }\end{array}$ & $\begin{array}{l}\text { OD:20/40 } \\
\text { OS:20/50 }\end{array}$ & Blurry vision & Blurry vision & No \\
\hline O4/F/63 & 2482 & a & SLE & 36 & $\begin{array}{l}\text { OD:20/25 } \\
\text { OS:20/25 }\end{array}$ & $\begin{array}{l}\text { OD:20/25 } \\
\text { OS:20/25 }\end{array}$ & Floaters & Flashes & Yes (OCT) \\
\hline O5/F/69 & 1550 & a & SLE & 12 & $\begin{array}{l}\text { OD:20/25 } \\
\text { OS:20/25 }\end{array}$ & $\begin{array}{l}\text { OD:20/25 } \\
\text { OS:20/25 }\end{array}$ & Glare & $\begin{array}{l}\text { Glare, float- } \\
\text { ers }\end{array}$ & No \\
\hline O6/F/67 & 1933 & 24 & SLE & 12 & $\begin{array}{l}\text { OD:20/25 } \\
\text { OS:20/25 }\end{array}$ & $\begin{array}{l}\text { OD:20/25 } \\
\text { OS:20/25 }\end{array}$ & None & None & Yes (OCT) \\
\hline O7/F/68 & 1048 & 26 & RA & 36 & $\begin{array}{l}\text { OD:20/20 } \\
\text { OS:20/30 }\end{array}$ & $\begin{array}{l}\text { OD:20/30 } \\
\text { OS:20/40 }\end{array}$ & None & $\begin{array}{l}\text { Decreased } \\
\text { near vision }\end{array}$ & Yes (OCT, FP) \\
\hline O8/F/75 & 2628 & 24 & RA & 12 & $\begin{array}{c}\text { OD:20/25 } \\
\text { OS:20/25 }\end{array}$ & $\begin{array}{l}\text { OD:20/25 } \\
\text { OS:20/25 }\end{array}$ & None & $\begin{array}{l}\text { Decreased } \\
\text { near vision }\end{array}$ & Yes (OCT) \\
\hline $\mathrm{S} 1 / \mathrm{F} / 63$ & 1030 & 23 & RA & 36 & $\begin{array}{l}\text { OD:20/400 } \\
\text { OS:20/100 }\end{array}$ & $\begin{array}{l}\text { OD:20/400 } \\
\text { OS:20/150 }\end{array}$ & $\begin{array}{l}\text { Decreased } \\
\text { central } \\
\text { vision }\end{array}$ & $\begin{array}{l}\text { Decreased } \\
\text { central } \\
\text { vision }\end{array}$ & Yes (OCT) \\
\hline$S 2 / F / 76$ & 1679 & 19 & RA & 14 & $\begin{array}{r}\text { OD:20/150 } \\
\text { OS:20/50 }\end{array}$ & $\begin{array}{l}\text { OD:20/100 } \\
\text { OS:20/100 }\end{array}$ & Blurry vision & Blurry vision & No \\
\hline
\end{tabular}

E2 left eye was included in Severe group analysis. E3 right eye was included in Obvious group analysis

a Information unavailable

SD-OCT data was available for all 30 eyes at the time of drug cessation and follow up examinations. The Early group comprised of eight eyes of 5 patients. The Obvious group comprised of 17 eyes of 9 patients. The Severe group comprised of 5 eyes of three patients. The right eye of patient E2 qualified for the Early group and the left eye for the Severe group. The right eye of patient E3 qualified for the Obvious group and the left eye for the Early group. All qualitative observations had a strong inter-observer correlation between the two observers from the reading center.

\section{SD-OCT at time of drug cessation}

Morphological appearance of 1-line horizontal SD-OCT scans in each of the groups at the time of drug cessation is shown in Fig. 2. In the fovea, $25 \%$ of the Early eyes and $59 \%$ of the Obvious eyes had clear disruption of the interdigitation zone (IZ) in the absence of ellipsoid zone
Table 2 Baseline characteristics of each group

\begin{tabular}{llll}
\hline & Early & Obvious & Severe \\
\hline Age (years) & $63(57-74)$ & $65(48-75)$ & $74(63-76)$ \\
$\begin{array}{l}\text { Cumulative dose } \\
\quad(g)\end{array}$ & $730(438-949)$ & $1933(584-4380)$ & $1030(890-1679)$ \\
Baseline vision & $20 / 20-20 / 40$ & $20 / 20-20 / 60$ & $20 / 100-20 / 400$ \\
Follow-up (months) & $13(12-56)$ & $36(12-56)$ & $14(13-36)$ \\
\hline
\end{tabular}

Median (range) reported for age, cumulative dose, and follow-up

disruption, suggesting that IZ disruption may precede EZ disruption. By our definition of the three groups, no patients in the Early or Obvious group had foveal disruption of the EZ while all patients in the Severe group did. In the Severe group, foveal external limiting membrane (ELM) disruption was observed in $40 \%$, while this 


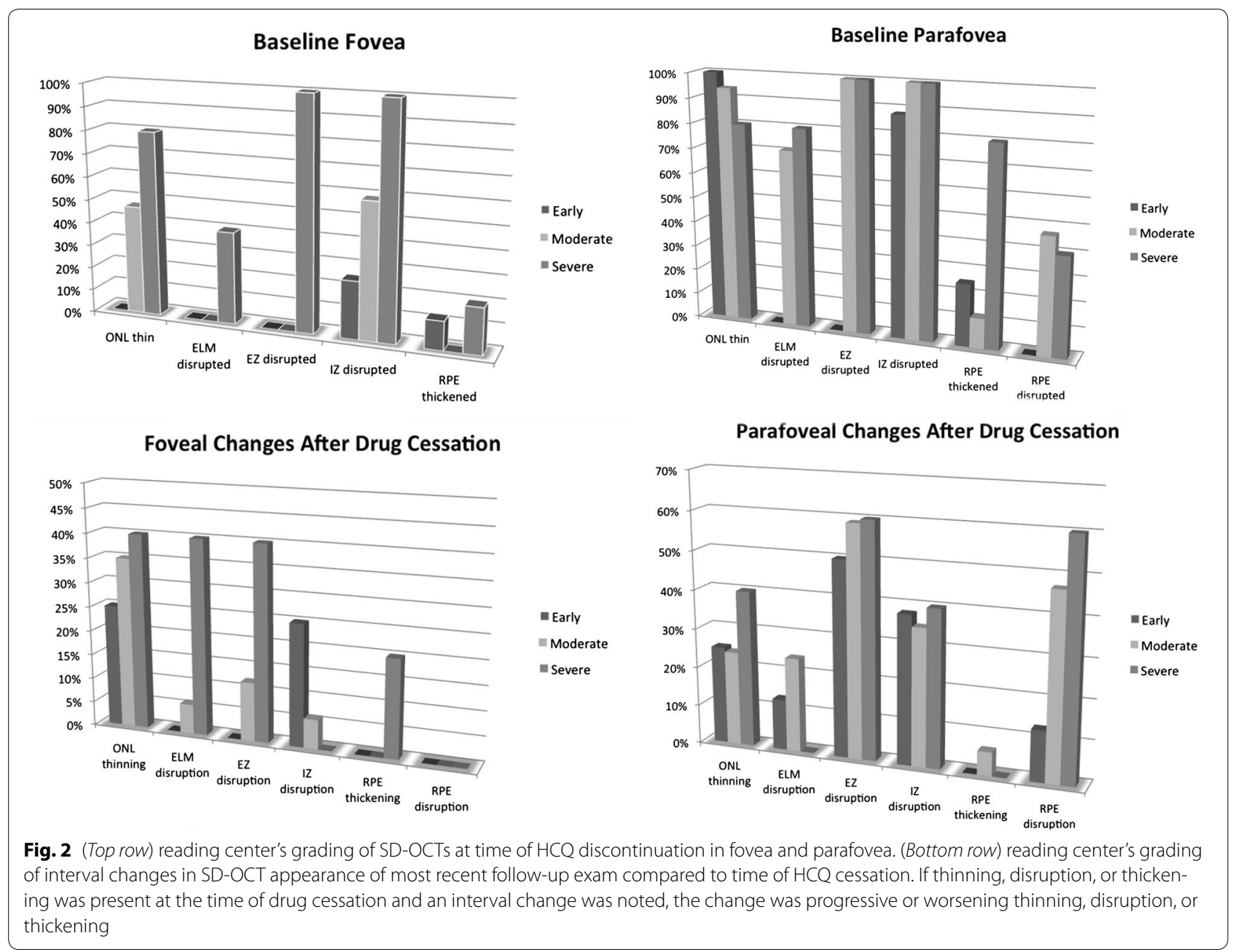

finding was not present in the other two groups. Foveal retinal pigment epithelium (RPE) disruption was not observed at baseline in any group. Foveal thinning of the outer nuclear layer $(\mathrm{ONL})$ was not present at baseline in any group.

In the parafoveal region, early SD-OCT findings were frequently detected in Early eyes in the absence of clear parafoveal EZ disruption (Fig. 3). Outer nuclear layer (ONL) thinning was present in $100 \%$ and observed as a focal indentation in the parafoveal ONL when compared to peripheral ONL thickness. Disruption of the parafoveal interdigitation zone (IZ) was recorded in $88 \%$ and reduced reflectivity of the parafoveal EZ band in the absence of definitive disruption was observed in $50 \%$. The reduced reflectivity of the parafoveal EZ enhanced the appearance of the foveal EZ, creating the illusion of increased prominence or hyperreflectivity of the foveal EZ (Fig. 4). Parafoveal RPE disruption was not detected in Early eyes, but was notable in $47 \%$ of Obvious eyes and $40 \%$ of Severe eyes.

\section{Progression of SD-OCT changes after drug cessation}

Morphological SD-OCT changes in each group after drug cessation is shown in Fig. 2. An interval change in SD-OCT appearance suggesting structural progression of toxicity was observed in $75 \%$ Early, $71 \%$ Obvious, and $100 \%$ of Severe eyes. In the Early group, 50 \% developed clear parafoveal EZ disruption after drug cessation with a median follow-up of 13 months (range 12-77 months; Fig. 5). Increased parafoveal EZ disruption was observed in $60 \%$ of the Obvious and Severe eyes. Increased parafoveal RPE disruption was detected in $13 \%$ Early eyes, $47 \%$ Obvious eyes, and $60 \%$ Severe eyes. There was no significant difference in the frequency or severity of temporal parafovea changes compared to the nasal parafovea. Foveally, the most common change seen on SD-OCT after drug cessation was progressive ONL thinning in $25 \%$ Early, 35 \% Obvious, and 40 \% Severe eyes. Progressive foveal RPE disruption was not detected. The latest time point in which progression was observed was progressive parafoveal RPE atrophy continuing for 4 years after drug 


\section{Early SD-OCT signs of HCQ toxicity before parafoveal ellipsoid zone (EZ) disruption:}

$\begin{array}{lr}\text { Parafoveal outer nuclear layer (ONL) thinning } & 100 \% \\ \text { Parafoveal interdigitation zone (IZ) disruption } & 88 \% \\ \text { Reduced reflectivity of parafoveal ellipsoid zone (EZ) } & 50 \%\end{array}$

Fig. 3 SD-OCT findings of early toxicity preceding the development of parafoveal EZ disruption

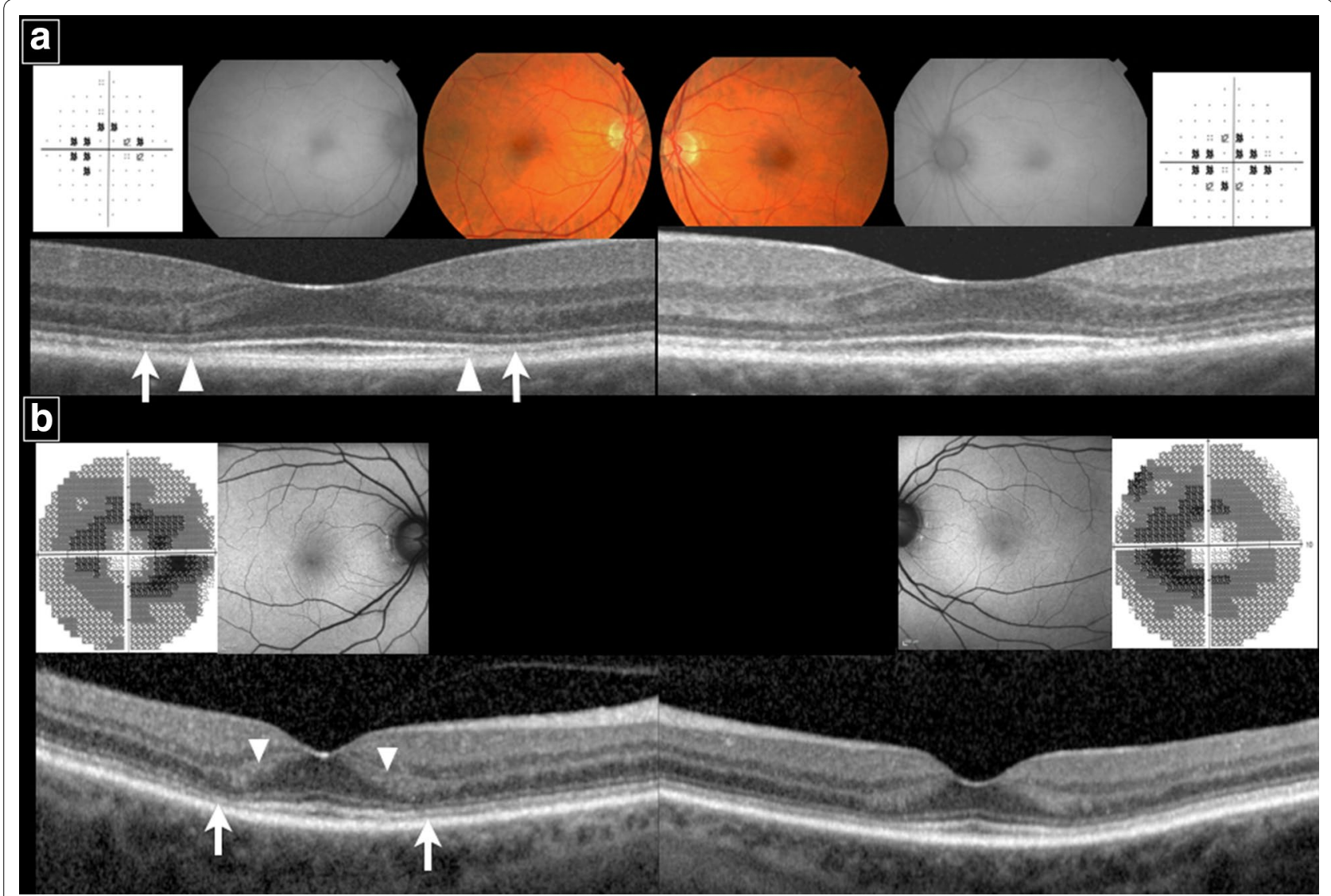

Fig. 4 Examples of two Early patients. a E1, fundus photograph shows mild parafoveal RPE changes in each eye with unremarkable FAF. HVF 10-2 shows incomplete parafoveal ring scotoma in right eye and parafoveal ring scotoma in left eye. SD-OCT of each eye shows no clear disruption of parafoveal EZ. However, reduced reflectivity of the parafoveal EZ (arrow) on both sides of the fovea is observed and enhances the appearance of the foveal EZ. Abrupt discontinuation of the parafoveal interdigitation zone (IZ) (arrowhead) is also observed on both sides of the fovea. b E4, Fundus autoflourescence is unremarkable. HVF 10-2 shows incomplete parafoveal ring scotomas in each eye. No clear disruption of the parafoveal EZ is seen on SD-OCT. However, the reflectivity of the parafoveal EZ is diminished (arrow) and enhances the appearance of the foveal EZ. There is also thinning of the parafoveal outer nuclear layer causing broadening of reflectivity of the parafoveal Henle's fiber layer (arrowhead) on both sides of the fovea

cessation (Fig. 6). Finally, a reduction in disruption or improvement in SD-OCT appearance was not observed.

\section{Macular thickness changes}

Figure 7 illustrates the change between HCQ cessation and 12 months after cessation in each of the 9 ETDRS
SD-OCT subfields. Decreases in central macular thicknesses were 3, 6, and $2 \mu \mathrm{m}$ in the Early, Obvious, and Severe groups, respectively. The largest reduction in ETDRS map region thickness was $7 \mu \mathrm{m}$ in the superior, nasal, and inferior outer rings of the Obvious group. When comparing thickness changes in the Obvious 


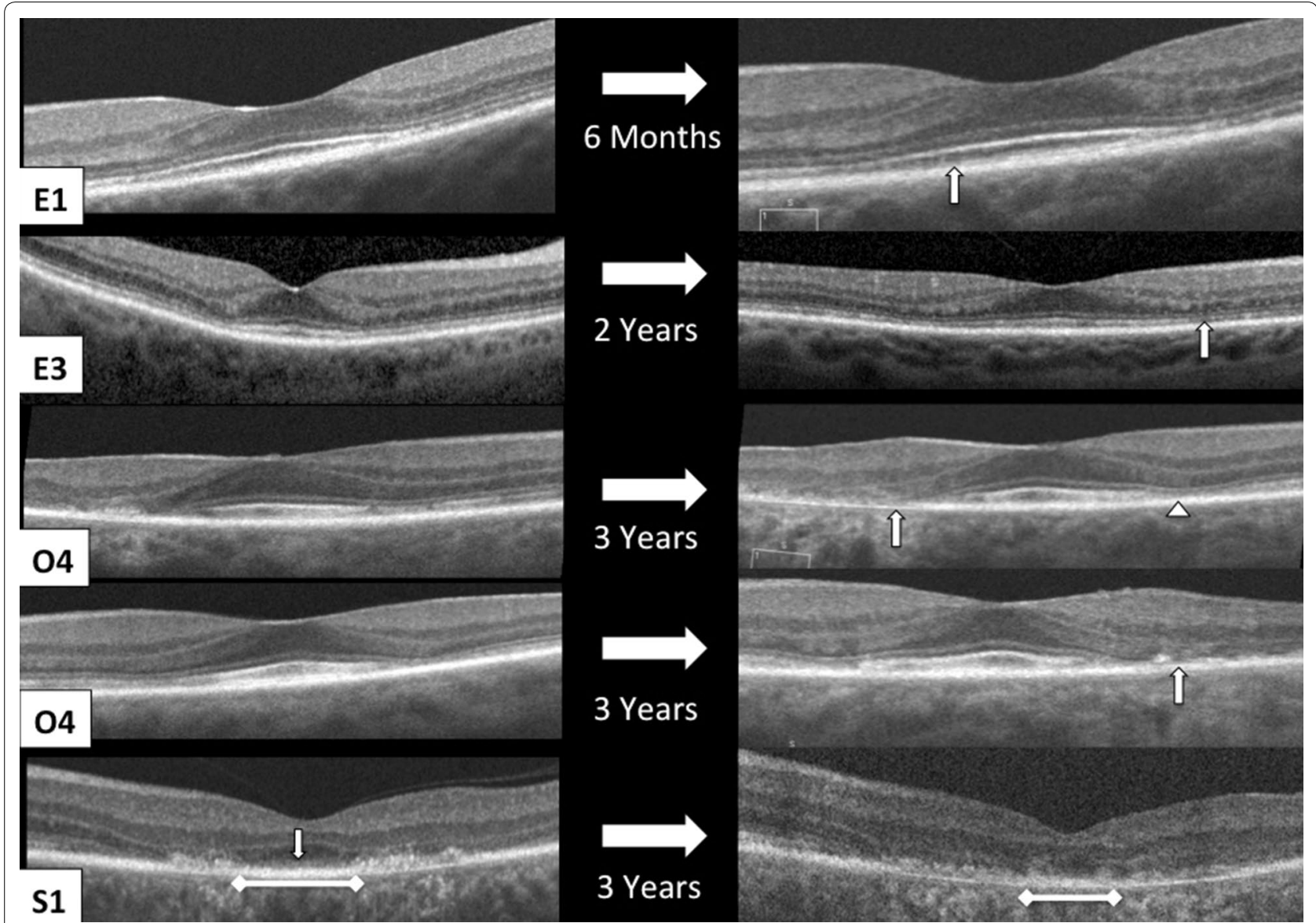

Fig. 5 Examples of changes in SD-OCT appearance from the time of diagnosis of HCQ retinopathy (left column) to after drug cessation (right column). E1, left eye, shows progressive interdigitation zone disruption (arrow). E4, right eye, shows development of parafoveal EZ disruption (arrow). O4, right eye, shows progressive RPE atrophy of the temporal parafovea (arrow) and progressive EZ disruption of the nasal parafovea (arrowhead). $\mathrm{O} 4$, left eye, shows progressive disruption of the ELM, EZ, and RPE in the temporal parafovea (arrow). S1, left eye, shows progressive loss of the foveal ELM (arrow), foveal RPE migration into outer retina, and disruption with shortening of foveal RPE (ruler)

group to the Early group, a significant difference was found in the inferior outer ring $(\mathrm{p}=0.002,95 \% \mathrm{CI}-2$ to $-8 \mu \mathrm{m})$. No differences between the groups were found in the other subfields. When comparing different subfield thickness changes within an individual group, no differences were observed within the Early or Severe groups. However in the Obvious group, the nasal inner subfield showed more thinning than the temporal inner subfield ( $\mathrm{p}=0.018,95 \% \mathrm{CI}-1$ to $-8 \mu \mathrm{m}$; Table 3 ).

A subanalysis of eyes with at least 3 years of follow-up after drug cessation ( $\mathrm{N}=11$ eyes; patients E3, E5, O2, $\mathrm{O} 3 \mathrm{OD}, \mathrm{O} 4, \mathrm{O} 7$; O3 OS excluded because of poor quality image at 42 month follow-up visit; S1 excluded because of widespread atrophy) was performed (Fig. 8). A progressive decline in retinal thickness beyond 1 year was observed in $62 \%$ of eyes. Median subfoveal thickness reduction was $14 \mu \mathrm{m}$. The largest decline in thickness was observed in the nasal inner ring $(15 \mu \mathrm{m})$. A difference in thickness change was not detected between nasal and temporal outer or inner rings $(\mathrm{p}=0.50 ; \mathrm{p}=0.11$, respectively) or superior and inferior outer and inner rings $(\mathrm{p}=0.60 ; \mathrm{p}=0.65$, respectively). In the Early patients, E3 developed thinning at 50 months compared to baseline, but had no follow-up visits in between. Therefore, it is uncertain at what time point the thinning occurred. Patient E5 showed thinning for the first 18 months after drug cessation but then the thickness increased between months 18 and 42 .

\section{Discussion}

By using masked, trained SD-OCT graders from the Boston Image Reading Center (BIRC), we determined that structural retinal changes were common after HCQ cessation, occurring in $73 \%$ of all eyes included in this 


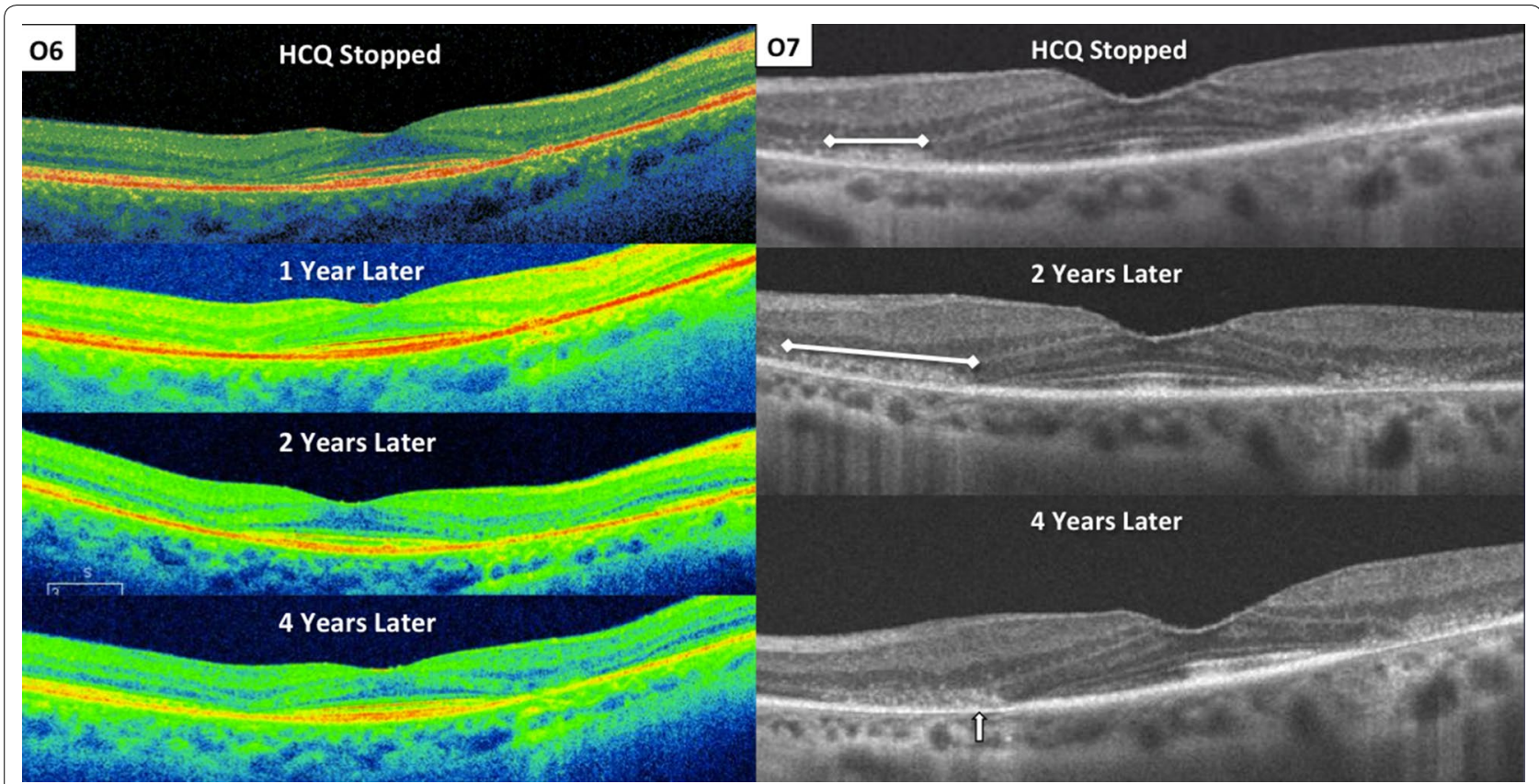

Fig. 6 SD-OCT changes 4 years after HCQ cessation. O6, right eye, shows development of parafoveal RPE atrophy that becomes evident 2 years after drug cessation and progresses at 4 year follow up. O7, left eye, shows progressive parafoveal RPE atrophy (arrow) with broadening of RPE migration into outer retina in the nasal parafovea (ruler)

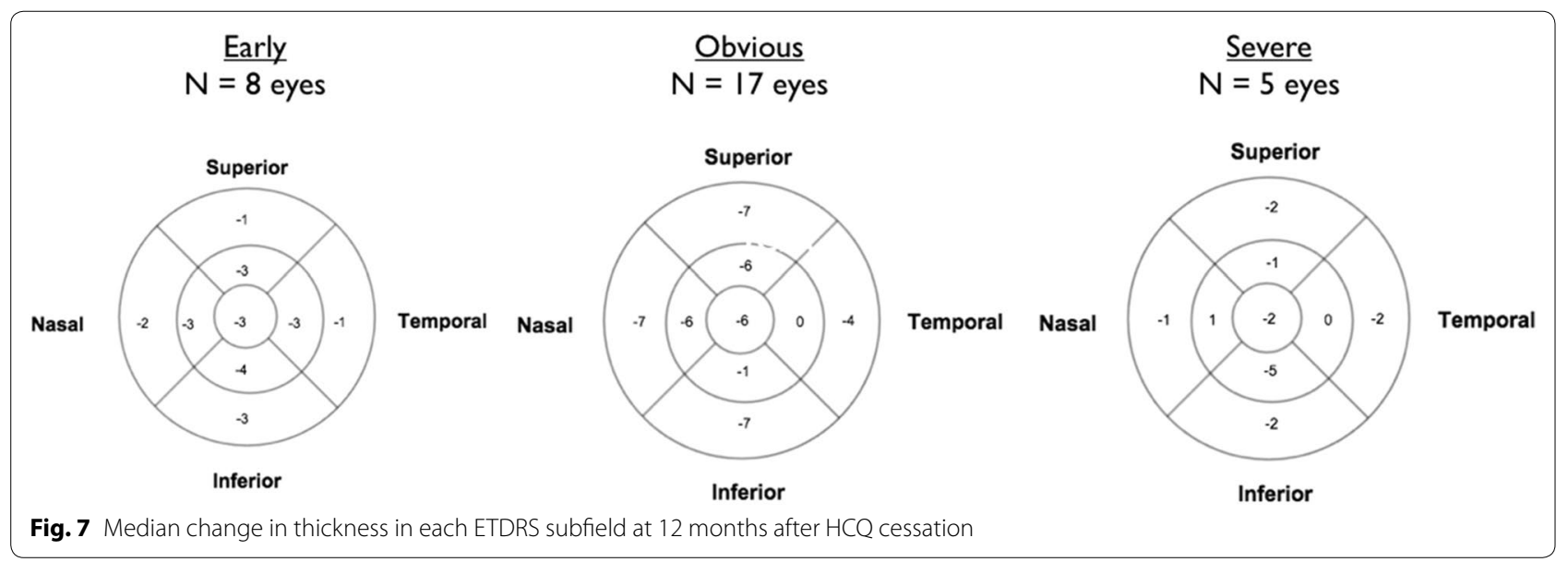

Table 3 P values for the comparison of ETDRS subfield thickness changes for Obvious group

\begin{tabular}{lcll}
\hline & Early & Obvious & Severe \\
\hline Nasal versus temporal inner & 1.000 & 0.018 & 0.660 \\
Superior versus inferior inner & 0.756 & 0.226 & 0.658 \\
Nasal versus temporal outer & 1.000 & 0.285 & 0.155 \\
Superior versus inferior outer & 0.626 & 0.291 & 0.264 \\
\hline
\end{tabular}

Italic value indicates significance of $p$ value $(p<0.05)$

$\mathrm{Cl} 95 \%$ confidence interval in micrometers study at a mean follow up of 26 months. All changes were consistent with progression of toxicity and may continue beyond 1 year most commonly as thinning of the parafoveal RPE (Fig. 6). In the Early group, 75 \% had evidence of structural progression after drug cessation, including disruption of the parafoveal EZ and RPE and thinning of the ONL (Table 1). These eyes developed structural progression despite presenting with subtle changes on SD-OCT at the time of HCQ cessation. Three of the five Early patients developed worsening visual field defects in 


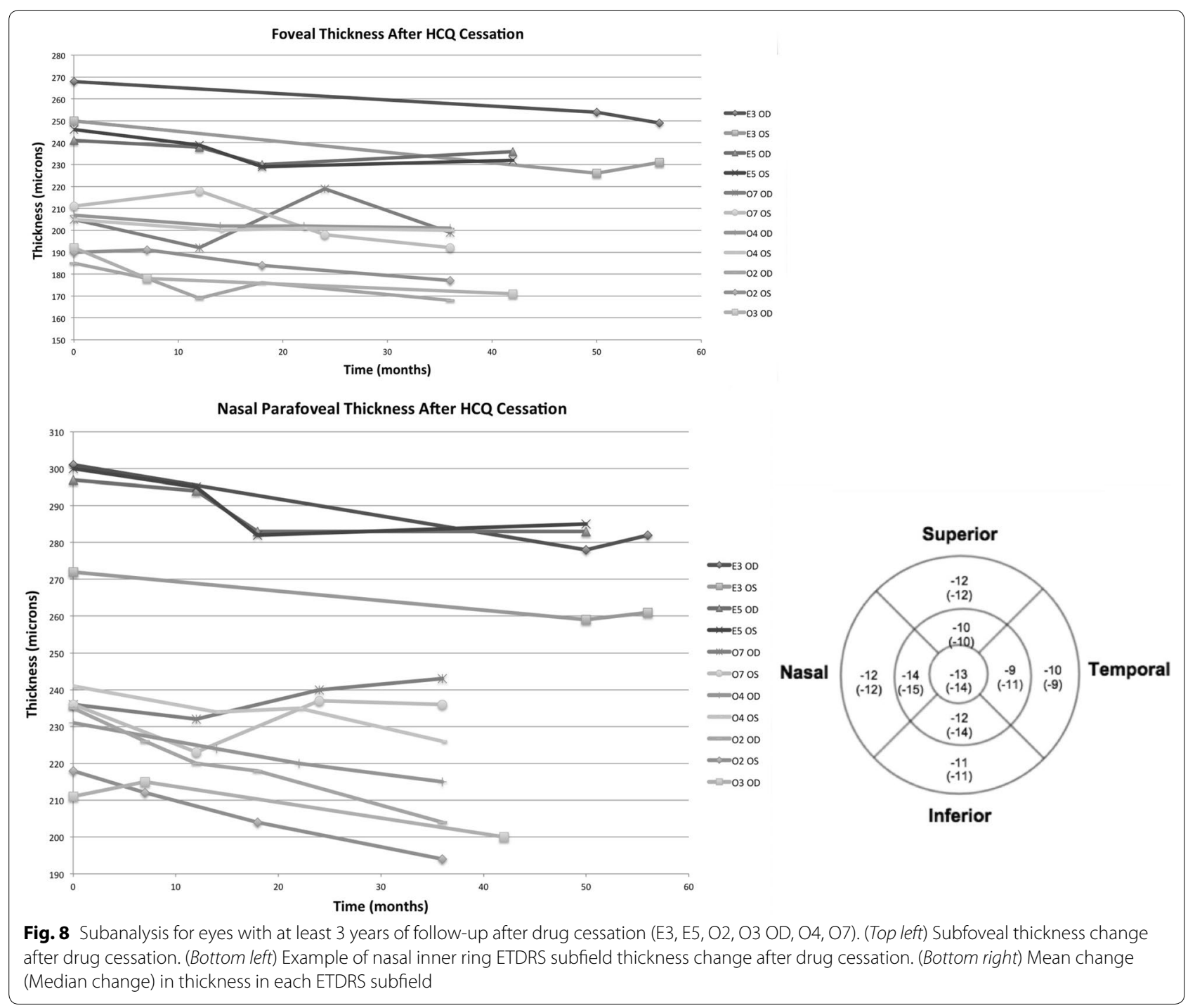

conjunction with structural changes. Importantly however, visual acuity and symptoms in these patients did not change significantly.

The disruption of the parafoveal ellipsoid zone is wellreported in HCQ toxicity, and clinicians commonly look for this finding in clinical practice [4, 11-13]. In this report, we describe three additional, clinically useful early SD-OCT signs of HCQ retinal toxicity that may precede disruption of the parafoveal ellipsoid zone (Fig. 3). We recommend looking carefully for these early findings to assist in the earliest detection of retinopathy. If an abnormality is detected, the authors recommend carefully checking the visual field searching for paracentral defects, as all Early eyes in this series had paracentral visual field defects at the time of drug cessation. This fact has been reported previously by Marmor and Melles [16] who described eyes with HCQ retinopathy in which the SD-OCT images were interpreted as normal but had paracentral visual field defects consistent with HCQ toxicity. Our review of these published images reveals that 8 of the 10 eyes displayed reduced reflectivity of the parafoveal EZ creating the appearance of a prominent foveal EZ band, suggesting that those eyes may have had reduced reflectivity of the parafoveal ellipsoid zone, as found in many of the Early eyes in our study. A criticism of this finding that we recognize is that the finding may be too subtle to be assuredly detectable in clinical practice. Often a 'normal' SD-OCT can have variance in scan brightness affecting EZ reflectivity. However, in these cases, the entire EZ band, including the foveal and parafoveal EZ, should have homogenous or near homogenous reflectivity. In our study, the masked graders were agreeable to the interpretation of EZ reflectivity, and therefore, we believe the reduced reflectivity of the parafoveal EZ is a real detectable SD-OCT finding in early HCQ 
toxicity. This sole finding should not be diagnostic of toxicity, but rather used to raise suspicion for toxicity and generate further investigation.

Disruption of the parafoveal interdigitation zone (IZ) was another common finding (88 \%) preceding parafoveal EZ disruption. The interdigitation zone, also known as the cone outer segment tip (COST) line, is the bright reflective line located between the EZ and the retinal pigment epithelium and is visible as a continuous line in $95 \%$ of normal subjects [17]. This finding has been reported in HCQ retinopathy two times previously, and appears to be another important but early finding in Early HCQ toxicity $[18,19]$.

Macular thickness changes in the Early and Severe groups were minimal at 12 months after drug cessation. Changes in the Severe group were presumed secondary to glial and RPE remodeling as these eyes had severe parafoveal and foveal outer retinal atrophy at the time of drug cessation. Obvious eyes had foveal thinning at 12 months similar to a previous report [11], and the macular thinning was not equal between the subfields. Greater thinning was detected in the nasal inner ring when compared to the temporal inner ring. This may be attributable to a thicker baseline nasal inner retina with more opportunity for thinning compared to the temporal inner retina. In addition, Obvious eyes developed greater thinning in the inferior outer ring as compared to Early eyes. This suggests an acceleration of thinning in the inferior outer ring as toxicity progresses. Interestingly, two other studies have noted the inferior macula may be the earliest affected quadrant in HCQ toxicity, but further studies are needed $[18,20]$. Finally, this study included myopic eyes up to $-6 \mathrm{D}$. The relationship between axial length and macular thickness measurements needs to be further clarified because axial length has both been negatively correlated to macular thickness but also not observed [21, 22]. Therefore, it remains unclear whether macular thickness changes in myopic eyes are different from emmetropic eyes.

Retained HCQ in the RPE cell may be the underlying cause of progressive retinal thinning observed in Obvious eyes after 12 months drug cessation. After intravenous injection of chloroquine into a rabbit, high concentrations of chloroquine are found in the pigmented ocular tissues (RPE, choroid, iris) [23]. These levels remain high for over 2 months following a single administration of chloroquine. Albino rabbits, on the other hand, do not retain chloroquine in the uvea or RPE indicating that melanin pigment is responsible for the binding and trapping of chloroquine within the cell. Once inside the RPE cell, HCQ disrupts lysosomal function leading to lipofuscin accumulation and photoreceptor degeneration [24]. We suspect HCQ is retained in the RPE cell for a prolonged period of time after drug cessation and may lead to progressive RPE atrophy, retinal thinning, and worsening visual field defects as observed in this study.

We did not observe improvement in visual acuity or visual fields after drug cessation. However, a study by Mititelu et al. [25] reported two patients with improvement in visual acuity and visual fields after HCQ cessation. The authors also observed parafoveal EZ regeneration and suggested the presence of an intact ELM at the time of drug cessation as a positive predictor for $\mathrm{EZ}$ regeneration. In contrast, EZ regeneration was not observed in our study, but progressive EZ disruption was noted in over half. Further studies are needed to support these findings.

SD-OCT, in combination with Humphrey's visual field $10-2$, as well as fundus autofluorescence and multifocal ERG in select patients, is critical for the early detection of HCQ retinopathy. As shown by this study and by Marmor and Melles [16], cases of early HCQ toxicity that display visual field changes attributable to HCQ toxicity in the setting of a 'normal SD-OCT' may in fact have early changes on SD-OCT as described in this report. We did not, however, observe any cases of HCQ retinopathy in which the visual field was normal but where morphological SD-OCT changes were detected. Therefore, a reliable visual field may be the most sensitive test for detecting early toxicity. However, fields are often unreliable, and SD-OCT has value in assisting detection in these cases. SD-OCT can also be used to corroborate early visual field defects. Conversely, if a parafoveal field defect is detected, we recommend repeating the field before deeming the finding unreliable.

\section{Conclusions}

SD-OCT can show signs of early HCQ retinopathy before unmistakable parafoveal EZ disruption develops, and these signs may assist in earlier detection of HCQ toxicity. Early detection is critical because both structural and functional progression may occur after the drug is discontinued.

\section{Abbreviations \\ SD-OCT: spectral domain-optical coherence tomography; FAF: fundus auto- fluorescence; mfERG: multifocal electroretinogram; HCQ: hydroxychloroquine;} RPE: retinal pigment epithelium; EZ: ellipsoid zone; ONL: outer nuclear layer.

\section{Authors' contributions}

DL participated in data gathering, data interpretation, and manuscript writing. SM participated in data interpretation and Table/Figure creation. IB participated in manuscript writing. JH, CB, AW, CS, ER, NW, AR, JD assisted in locating patients who fulfilled inclusion criteria as well as critical revision of the manuscript. All authors read and approved the final manuscript.

\section{Author details}

${ }^{1}$ Vitreoretinal Service, Departments of Ophthalmology, New England Eye Center, Tufts University School of Medicine, 260 Tremont Street, Boston, MA 02116, USA. ${ }^{2}$ Vitreoretinal Service, Ophthalmic Consultants of Boston, Boston, MA, USA. 


\section{Acknowledgements}

David R. Lally, MD had full access to all the data in the study and takes responsibility for the integrity of the data and accuracy of the data analysis.

\section{Competing interests}

The authors declare that they have no competing interests.

\section{Availability of data}

The raw data will not be made available because it is possessed by the reading center.

\section{Consent for publication}

All subjects in the study gave permission for the images and information shown within the manuscript.

\section{Ethics approval and consent to participate}

Data collection was obtained following approval from the Internal Review Board (IRB) of Tufts Medical Center with IRB reference \#11103, and was in compliance with the Health Insurance Portability and Accountability Act. Research tenets were followed in accordance with the Declaration of Helsinki.

\section{Financial disclosures}

Dr. Jay Duker is a consultant and receives research support from Carl Zeiss Meditech. Drs. Lally, Heier, Witkin, Baumal, Reichel, Shah, Maler, Waheed, Bussel, Rogers do not have financial disclosures relevant to this paper.

\section{Funding/support}

This work was supported in part by Ophthalmic Consultants of Boston's CERE account and an unrestricted grant from the Research to Prevent Blindness to the New England Eye Center, Tufts University School of Medicine and the Massachusetts Lions Clubs.

Received: 8 April 2016 Accepted: 21 June 2016

Published online: 18 July 2016

\section{References}

1. Rynes RI. Antimalarial drugs in the treatment of rheumatological diseases. Rheumatology. 1997;36(7):799-805.

2. Shearer RV, Dubois EL. Ocular changes induced by long-term hydroxychloroquine (plaquenil) therapy. Am J Ophthalmol. 1967;64:245-52.

3. Wolfe F, Marmor MF. Rates and predictors of hydroxychloroquine retinal toxicity in patients with rheumatoid arthritis and systemic lupus erythematosus. Arthritis Care Res (Hoboken). 2010;62:775-84.

4. Marmor MF, Kellner U, Lai TTY, for the American Academy of Ophthalmology, et al. Revised recommendations on screening for chloroquine and hydroxychloroquine retinopathy. Ophthalmology. 2011:118:415-22.

5. Carr RE, Henkind P, Rothfield N, et al. Ocular toxicity of antimalarial drugs: long-term follow-up. Am J Ophthalmol. 1968;66:738-44.

6. Brinkley JR Jr, Dubois EL, Ryan SJ. Long-term course of chloroquine retinopathy after cessation of medicine. Am J Ophthalmol. 1979;88:1-11.

7. Ogawa S, Kurumatani N, Shibaike N, Yamazoe S. Progression of retinopathy long after cessation of chloroquine retinopathy. Lancet. 1979;1:1408
8. Maturi RK, Yu M, Weleber RG. Multifocal electroretinographic evaluation of long-term hydroxychloroquine users. Arch Ophthalmol. 2004;122:973-81.

9. Lai TTY, Chan WM, Li H, et al. Multifocal electroretinographic changes in patients receiving hydroxychloroquine therapy. Am J Ophthalmol. 2005; 140:794-807.

10. Kellner U, Renner AB, Tillack H. Fundus autofluorescence and mfERG for early detection of retinal alterations in patients using chloroquine/ hydroxychloroquine. Investig Ophthalmol Vis Sci. 2006;47:3531-8.

11. Marmor M, Hu J. Effect of disease stage on progression of hydroxychloroquine retinopathy. JAMA Ophthalmol. 2014;132:1105-12.

12. Rodriguez-Padilla JA, Hedges TR, Monson B, et al. High-speed ultra-high resolution optical coherence tomography findings in hydroxychloroquine retinopathy. Arch Ophthalmol. 2007;125:775-80.

13. Chen E, Brown DM, Benz MS, et al. Spectral domain optical coherence tomography as an effective screening test for hydroxychloroquine retinopathy (the "flying saucer" sign). Clin Ophthalmol. 2010;21:1151-8.

14. Pasadhika S, Fishman GA, Choi D, Shahidi M. Selective thinning of the perifoveal inner retina as an early sign of hydroxychloroquine retinal toxicity. Eye. 2010;24:756-63.

15. Kellner S, Weinitz S, Farmand G, et al. Cystoid macular oedema and epiretinal membrane formation during progression of chloroquine retinopathy after drug cessation. Br J Ophthalmol. 2014;98:200-6.

16. Marmor M, Melles RB. Disparity between visual fields and optical coherence tomography in hydroxychloroquine retinopathy. Ophthalmology. 2014;121:1257-62.

17. Rii T, Itoh $Y$, Inoue $M$, et al. Foveal cone outer segment tips line and disruption artifacts in spectral-domain optical coherence tomographic images of normal eyes. Am J Ophthalmol. 2012;153:524-9.

18. Marmor M. Comparison of screening procedures in hydroxychloroquine toxicity. Arch Ophthalmol. 2012;130:461-9.

19. Barnes AC, Bhavsar K, Weber ML, Witkin AJ. A early case of hydroxychloroquine retinopathy: spectral domain optical coherence tomography findings. Eye. 2014;28:1521-2.

20. Ulviye $\mathrm{Y}$, Betul T, Nur TH, et al. Spectral domain optical coherence tomography for early detection of retinal alterations in patients using hydroxychloroquine. Indian J Ophthalmol. 2013;61:168-71.

21. Song AP, Wu XY, Wang JR, Liu W, Sun Y, Yu T. Measurement of retinal thickness in macular region of high myopic eyes using spectral domain OCT. Int J Ophthalmol. 2014;7:122-7.

22. Zhao MH, Wu Q, Hu P, Jia LL. Macular thickness in myopia: an OCT study of young Chinese patients. Curr Eye Res 2016;10:1-6 [Epub ahead of print].

23. Bernstein $\mathrm{H}$, Zvaifler N, Rubin M, Mansour AM. The ocular deposition of chloroquine. Investig Ophthalmol. 1963;2:384-92.

24. Sundelin SP, Terman A. Different effects of chloroquine and hydroxychloroquine on lysosomal function in cultured retinal pigment epithelial cells. APMIS. 2002;110:481-9.

25. Mititelu M, Wong BJ, Brenner $M$, et al. Progression of hydroxchloroquine toxic effects after drug therapy cessation: new evidence from multimodal imaging. JAMA Ophthalmol. 2013;131:1187-97.

\section{Submit your next manuscript to BioMed Central and we will help you at every step:}

- We accept pre-submission inquiries

- Our selector tool helps you to find the most relevant journal

- We provide round the clock customer support

- Convenient online submission

- Thorough peer review

- Inclusion in PubMed and all major indexing services

- Maximum visibility for your research

Submit your manuscript at www.biomedcentral.com/submit 